\title{
Duct azimuthal and radial modal detection on the CFD modeling of UHBR engine tonal noise.
}

\author{
S. Fauqueux* \\ French Aerospace Lab (ONERA), Aerodynamics Aeroelasticity Acoustics Department, Avenue de la Division Leclerc, \\ 92322 Châtillon Cedex, France
}

\begin{abstract}
Modal deconvolution method is used to characterize the main acoustic modes propagating in duct from non-intrusive measurements. In the framework of the ASPIRE-CS2 project, ONERA's modal deconvolution method ARMADA is applied to numerical data from a generic UHBR engine at the take-off sideline condition. The numerical simulation is provided by NLR to quantify the tonal internal acoustic field by the application of CFD approach for the configuration with clean fan channel. The complex geometry of the nacelle implies a variable Mach number through the fan duct, which is unfortunately not precisely known.

As a first step, azimuthal Fourier transform is applied to determine the dominant azimuthal modes content. In the bypass duct (downstream direction), they are shown to be quite constant, whereas, upstream from the fan, they sharply vary in the vicinity of the inlet. Therefore, our deconvolution method is only applied on the bypass duct, using data obtained at BPF2 on the wall as it would be for flush mounted microphones. The modal basis is restricted to azimuthal mode orders from -1 to 11 , which is relevant to explain the main content of the total sound pressure. Considering a constant flow rate with two hypotheses of Mach number (0.4 and 0.5$)$, and under the correlated mode assumption, the results explain more than $74 \%$ of the data and dominant modes are found to be perfectly correlated. Modes propagating in the upstream direction point out possible reflections at the end of the nacelle. Finally, the acoustic pressure field is reconstructed from the estimated modes, and compared to the initial numerical data. Using only data obtained on the wall, ONERA's deconvolution method succeeds in providing a good representation of the acoustic field in the bypass duct.
\end{abstract}

\section{Introduction}

The reduction of noise pollution caused by aircraft is one of the major concern of the aerospace industry. This reduction is essential in the take-off and landing phases, generally operated in densely populated areas. Two types of noise pollution occur during flight : the aerodynamic noise due to the displacement of the aircraft in the air, and the noise generated by the propulsion unit. The latter is composed of the jet noise associated with the turbulence generated by the ejection of the air at the outlet of the nozzle, as well as fan and turbine noise, and those from the compressor and the combustion chamber. Although noise appears in all phases of flight, it is clear that the one due to propulsion is dominant during the take-off phase. The increase in the dilution ratio between the primary and secondary flow, combined with the use of acoustic absorbing materials, allows a decrease in jet noise. The evolution towards engines with very high by-path ratio tends to make the fan noise predominant compared to jet noise, and has to be reduced in the coming years consequently. This reduction goes through the identification and characterization of the main sources, in order to develop specific treatments, by using non-intrusive experimental antenna.

ONERA has recently developed a modal deconvolution method, called ARMADA [1], based on the iterative minimization of the discrepancy, in the least-square sense, between flush-mounted microphone measurements and an analytical model that represents the propagation between the sources and the measurements positions. In the framework of the ASPIRE-CS2 project, NLR has quantified the tonal noise sources of a generic UHBR engine by the application of CFD technology, solving the time-dependent Reynolds-Averaged Navier-Stokes equations while the fan advances in small azimuthal steps [2]. Our aim is to apply our deconvolution method on data obtained at the take-off condition, for the configuration with a "clean" fan exhaust duct geometry (i.e. without bifurcations). ARMADA's analytical model assumes a rigid and infinite cylindrical or annular duct of constant radius submitted to a uniform and subsonic flow along the revolution axis of the duct. The configuration of interest implies a highly variable Mach number through the

\footnotetext{
*Sandrine.Fauqueux@onera.fr.
} 
fan channel, due to a complex geometry of the nacelle. Therefore, it would be necessary to reduce the study domain (geometrical part of the nacelle and/or frequency) so that the hypotheses underlying our method stay valid.

To do so, different methods are applied, step by step, from simple to increasingly complexity. Analysis is performed on the time Fourier transform of the acoustic pressure field evaluated on the inlet and bypass duct surfaces, as our deconvolution method would be applied to non intrusive measurements (flush mounted microphones). First of all, we analyse the azimuthal mode content of the acoustic pressure field by using an azimuthal Fourier Transform. This will also help us to determine a domain (i.e. part of the nacelle) where the azimuthal mode content is relatively constant. We focus on data provided on bypass duct at BPF2. Only few azimuthal modes are relevant to explain the main content of the total sound pressure. An azimuthal mode deconvolution method is then applied, assuming that modes are correlated or not. If azimuthal modes are uncorrelated, our problem will be simplified by making a radial deconvolution method separately on each azimuthal mode order. If not, it would be necessary to take into account all the correlations : between azimuthal as well as radial modes. Finally, we apply our azimuthal and radial mode deconvolution method ARMADA.

\section{Azimuthal mode analysis by Fourier transform}

As a first step, an azimuthal analysis is performed, based on azimuthal Fourier transform. We introduce the cylindrical coordinates system associated to the orthonormal coordinates system $(X, Y, Z)$ of the nacelle geometry (see Figure 11. $X=0$ corresponds to the axial fan position. The time Fourier transform of the acoustic pressure field is expressed as a sum of weighted azimuthal modes $e^{-\mathrm{i} m \theta}$. The azimuthal mode base forms an orthogonal basis when considering the scalar product defined by the integration, over $[0,2 \pi]$, of the cross product of the functions. Then, one can write :

$$
\left|\frac{1}{2 \pi} \int_{0}^{2 \pi} \hat{p}\left(X_{0}, R, \theta\right) \times e^{\mathrm{i} m \theta} d \theta\right|=\left|A_{m}\left(X_{0}, R\right)\right|
$$

where $A_{m}\left(X_{0}, R\right)$ is the amplitude of the azimuthal mode $m$ at section $X=X_{0}$ and radial position $R$. In practice, we only consider $R$ as the hub and casing radius.
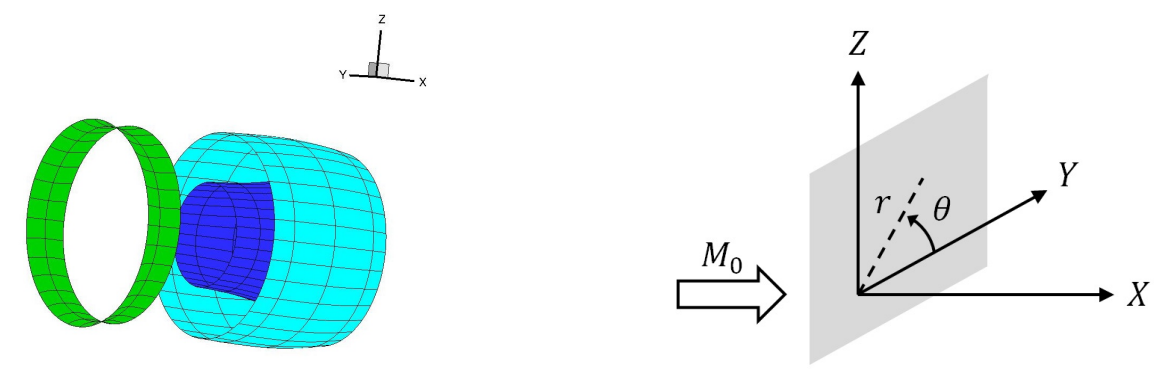

Fig. 1 Inlet and bypass duct surfaces (left) and cylindrical and orthonormal coordinates systems (right).

The bypass duct rotation axis corresponds to the $X$-axis, whereas the inlet duct axis is lightly curved, closed to the air intake. Thus, the polar coordinates of the axial section of the duct wall may be defined as the polar coordinates of an $Y=Z=0$ centred circle closed to the cross section, or of the circle that fit the best the cross section. In practice, there is no significant impact on the results. The amplitude of azimuthal modes, normalized by its maximum value and obtained at BPF2 in the inlet and bypass duct casing, are represented in figure 2 At the inlet duct casing, the rotor-locked mode is dominant closed to the fan but scatter in the vicinity of the inlet due to distortion and high variation of the flow, as illustrated in [2] (Figure 5.). The bypass duct casing and hub, whose azimuthal analysis results are not represented, show a more constant azimuthal mode content where the first interaction mode is dominant. Furthermore, only few azimuthal modes explain the most part of the total sound pressure level, especially if we restrict ourselves to section from $X=1.57$ $\mathrm{m}$ to $2.9 \mathrm{~m}$, where azimuthal modes from -1 to 11 explains more than $98 \%$ of the total sound pressure level.

\section{Azimuthal mode deconvolution at BPF2 on the bypass duct}

As a second step, an azimuthal deconvolution method is applied on the acoustic pressure field computed at BPF2 in the bypass duct casing and hub separately (no radial dependence). The deconvolution analysis is based on ONERA's 

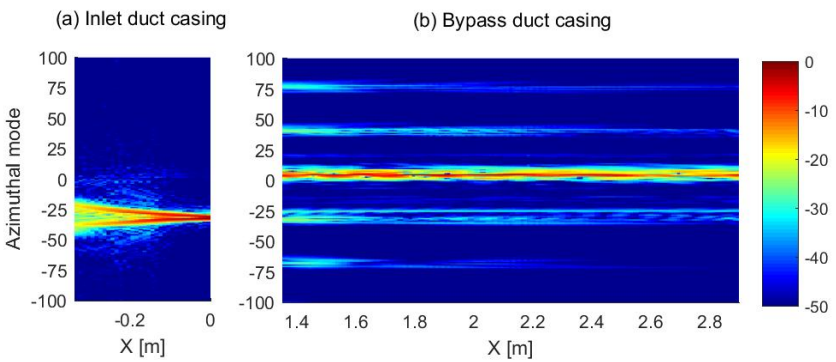

Fig. 2 Normalized amplitude of azimuthal modes, in dB, obtained at BPF2 in the inlet (a) and bypass (b) duct casing.

method ARMADA [1], considering a modal basis only composed by azimuthal modes of order from -1 to 11 . The problem to solve is expressed as :

$$
\tilde{\mathbf{S}}^{[m]}\left(X_{0}\right)=\arg \min _{\mathbf{S}^{[m]}}\left\|\boldsymbol{\Gamma}^{\text {mesure }}\left(X_{0}\right)-\mathbf{G}^{[m]} \mathbf{S}^{[m]} \mathbf{G}^{\mathrm{H}[m]}\right\|_{\mathrm{F}}^{2}
$$

with $\|\cdot\|_{\mathrm{F}}$ the Frobenius matrix norm. $\Gamma^{\text {mesure }}\left(X_{0}\right)$ denotes the cross-spectral matrix of measurements obtained on a virtual azimuthal microphones ring located at $X=X_{0} . \mathbf{S}^{[m]}\left(X_{0}\right)$, defined as :

$$
\mathbf{S}_{i, j}^{[m]}\left(X_{0}\right)=\overline{A_{m_{i}}\left(X_{0}, R\right) A_{m_{j}}^{*}\left(X_{0}, R\right)}
$$

where $z^{*}$ is the complex conjugate of $z$ and $\bar{z}$ denotes the temporal expected value. It has to be an Hermitian, positive and semi-definite matrix. $\mathbf{G}^{[m]}$ is defined by :

$$
\mathbf{G}_{i, j}^{[m]}=e^{-\mathrm{i} m_{i} \theta_{j}}
$$

and we restrict ourselves to $-1 \leq m_{i} \leq 11$.

Modes are assumed to be correlated or not. Under the uncorrelated mode assumption, the unknown matrix $\mathbf{S}^{[m]}$ is diagonal. $\mathbf{S}^{[m]}$ is a full matrix otherwise. The diagonal part of $\mathbf{S}^{[m]}$ contains the square amplitudes of the azimuthal modes. The virtual microphone array is composed by a single ring of 23 microphones regularly spaced. The problem (2) is iteratively resolved by using the same algorithm as in our azimuthal and radial mode deconvolution method ARMADA (see [1] for more details).

First of all, the amplitude of the most energetic azimuthal modes obtained with the uncorrelated and the correlated azimuthal deconvolution methods are exactly the same. They are, thus, compared with the one obtained by the azimuthal Fourier Transform. The gap is always smaller than $0.6 \mathrm{~dB}$ when analysing data from the bypass duct casing surface, except at $X=1.6 \mathrm{~m}$ where a discrepancy of $1.03 \mathrm{~dB}$ for azimuthal mode of order 3 is noticed (see Figure 3 ). For data from the bypass duct hub surface, the discrepancy is always smaller than $0.24 \mathrm{~dB}$ (see Figure 4). One can also notice that the azimuthal mode content of the pressure field varies along the $X$-axis and is different in the hub and casing ducts.
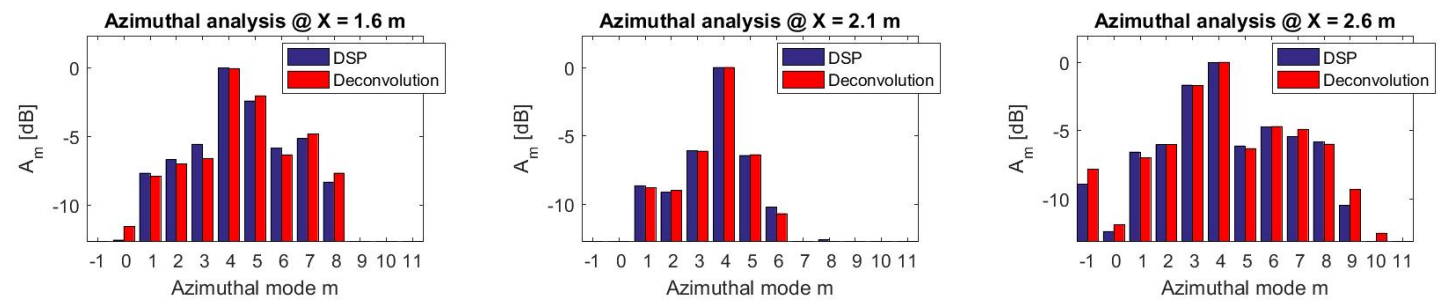

Fig. 3 Relative azimuthal mode amplitudes [dB] obtained with the azimuthal Fourier transform (blue) and deconvolution methods (red) at different sections of the bypass duct casing.

However, the part of the power cross spectral matrix of the data that is explained by the azimuthal Fourier

\footnotetext{
*Defined by : $100 \times\left[1-\frac{\| \boldsymbol{\Gamma}^{\text {mesure }}\left(X_{0}\right)-\mathbf{G}^{[m]} \tilde{\mathbf{S}}^{[m]} \mathbf{G}^{\mathrm{H}}}{\left\|\boldsymbol{\Gamma}^{\text {mesure }}\left(X_{0}\right)\right\|_{\mathrm{F}}} \|_{\mathrm{F}}\right]$ with $\tilde{\mathbf{S}}^{[m]}$ obtained by the different methods.
} 

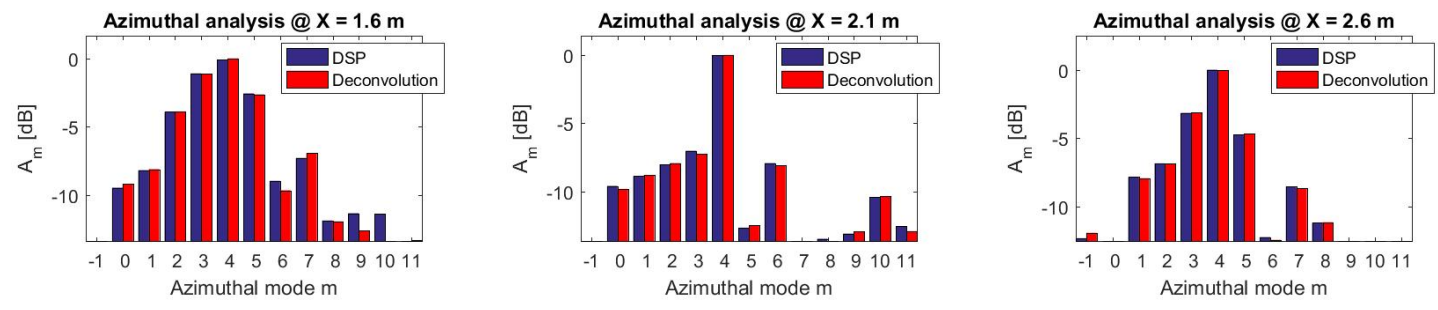

Fig. 4 Relative azimuthal mode amplitudes [dB] obtained with azimuthal Fourier transform (blue) and deconvolution methods (red) at different sections of the bypass duct hub.

transform and the uncorrelated azimuthal deconvolution method is quite low, whereas results obtained with the azimuthal deconvolution method under correlated modes assumption explains the most part of the data (see Figure 5). This means that the extra-diagonal part of the power cross-spectral matrix of the amplitude of azimuthal modes can not be neglected.
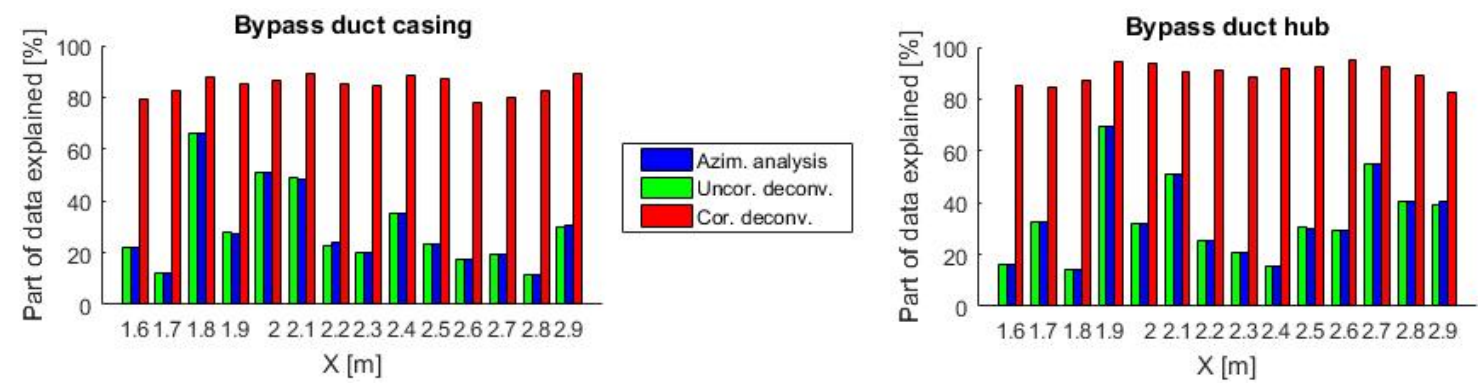

Fig. 5 Part of the data explained by the power cross spectral matrix of the amplitude of the azimuthal modes estimated with the azimuthal Fourier transform (blue), the uncorrelated (green) and the correlated (red) azimuthal deconvolution methods at different sections of the bypass duct casing (left) and hub (right).

Even if data are better explained under the correlated than the uncorrelated modes assumption, non-zero correlations may been caused by numerical artefacts, as the number of unknowns to estimate is greater under the first than under the second hypothesis. However, the estimated cross spectral matrix has to be an Hermitian, positive and semi-definite matrix. Since only few azimuthal modes have significant amplitudes, the number of extra-diagonal terms with potentially significant value is reduced. Considering the bypass duct casing cross-section at $X=2.6 \mathrm{~m}$, the extra-diagonal part of the azimuthal cross spectral matrix obtained when considering azimuthal modes varying from -100 to 100 and azimuthal modes varying from -1 to 11 are exactly the same. The emergence of numerical artefacts seems unlikely. Another reason can explain the azimuthal mode correlations : the mode content of the acoustic pressure field, composed by Tyler-Sofrin interaction and adjacent modes, changes along the $X$-axis, probably caused by variation in the geometry as well as in the flow. Thus, as they all are induced by the same physical phenomenon, those modes may be correlated. Those results encourage us to consider that azimuthal modes are correlated and, then, apply our azimuthal and radial deconvolution method ARMADA under the correlated modes assumption.

\section{Azimuthal and radial mode deconvolution at BPF2 on the bypass duct}

The deconvolution method [1] is extended to annular duct. The Fourier transform of the acoustic pressure field propagating inside a rigid infinite annular duct of constant external radius $R$ and constant internal to external radius ratio $h$, in the presence of a uniform subsonic flow along the revolution-axis of the duct, is expressed as [3] :

$$
\hat{p}(X, r, \theta, v)=\sum_{m=-\infty}^{+\infty} \sum_{n=0}^{n=+\infty} \sum_{\varepsilon= \pm} A_{m, n}^{\varepsilon}(v) \times U_{m, n}(r) e^{-\mathrm{i} m \theta} e^{-\mathrm{i} k_{m, n}^{\varepsilon}(v) X}
$$


where $m \in \mathbb{Z}$ and $n \in \mathbb{N}$ are called the azimuthal mode and radial mode respectively and $A_{m, n}^{\varepsilon}$ is the mode amplitude. $U_{m, n}$ is a linear combination of $m$-th order Bessel functions of the first and of the second kind, that depends on $h$ and $R$. $k_{m, n}^{\mathcal{E}}(v)$ is given by the dispersion relation, and depends on the Mach number $M_{0}$, the speed of sound, the azimuthal and radial mode orders as well as the frequency $v$ and the geometry $(h$ and $R$ ). In practice, the sum is finite as only cut-on modes are considered. Let us introduce $N_{\text {mode }}$ as the number of cut-on modes at the frequency $v . k_{m, n}^{+}$and $k_{m, n}^{-}$are related to waves propagating downstream and upstream respectively. In the following, $v$ is omitted.

We consider a virtual antenna, composed by $N$ flush-mounted microphones of coordinates $\left(X_{i}, \theta_{i}, R_{i}\right)_{i=1 . . N}$, where $R_{i}$ takes the value $R$ or $h \times R$ exclusively. The mode amplitudes are estimated by solving the following problem, similar to the one given by equation $(2)$ :

$$
\tilde{\mathbf{S}}=\arg \min _{\mathbf{S}}\left\|\boldsymbol{\Gamma}^{\text {mesure }}-\mathbf{G} \mathbf{S ~ G} \mathbf{G}^{\mathrm{H}}\right\|_{\mathrm{F}}^{2}
$$

with $\|\cdot\|_{F}$ the Frobenius matrix norm. $\Gamma^{\text {mesure }}$ denotes the power cross-spectral matrix of the acoustic pressure field computed at the microphones of the virtual antenna that has to be defined. $\mathbf{S}$, is defined as :

$$
\mathbf{S}_{i, j}=\overline{A_{m_{i}, n_{i}}^{\varepsilon_{i}} A_{m_{j}, n_{j}}^{\varepsilon_{j} *}}
$$

and has to be an Hermitian, positive-semi definite matrix. $\mathbf{G}$ is defined by :

$$
\mathbf{G}_{i, j}=U_{m_{i}, n_{i}}\left(R_{j}\right) e^{-\mathrm{i} m_{i} \theta_{j}} e^{-\mathrm{i} k_{m_{i}, n_{i}}^{\varepsilon_{i}} X_{j}}
$$

The under-constraint minimization problem (6) is resolved by the same iteration algorithm as presented in [1]. The

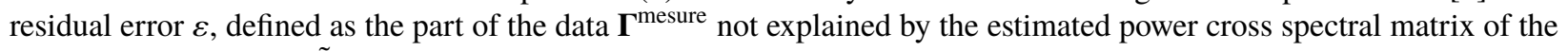
amplitude of the mode $\tilde{\mathbf{S}}$ is evaluated :

$$
\varepsilon=\frac{\left\|\boldsymbol{\Gamma}^{\text {mesure }}-\mathbf{G} \tilde{\mathbf{S}} \mathbf{G}^{\mathrm{H}}\right\|_{\mathrm{F}}}{\left\|\boldsymbol{\Gamma}^{\text {mesure }}\right\|_{\mathrm{F}}} \times 100
$$

The expression given by equation (5) is valid for acoustic field propagating in infinite rigid annular duct of constant section, submitted to a uniform flow. The case that is investigated in this article significantly differs from that simplified configuration : the inlet and bypass duct radius vary along the $X$-axis and the flow is non-uniform. We assume that the bypass duct flow rate is constant and that the modal basis associated to each infinitesimal section $X$ is equivalent to the one obtained with an infinite annular duct of outer radius $R_{\text {ext }}(X)$ and inner radius $R_{\text {int }}(X)$ submitted to a uniform flow. The Mach number varies through the fan duct and is unfortunately not precisely known. Two values will be taken in the rest of the article : 0.4 or 0.5 at $X=1.3 \mathrm{~m}$. As for azimuthal deconvolution, we only consider azimuthal modes order varying from -1 to 11 . $\left(k_{m_{i}, n_{i}}^{\varepsilon_{i}}(X)\right)_{i=1 \ldots N_{\text {mode }}}$ evaluated with a Mach number equal to 0.4 at $X=1.3 \mathrm{~m}$ are represented in Figure 6. Wave numbers are varying, especially from $X=1.6$ to $2.02 \mathrm{~m}$, where some modes go from cut-on to cut-off. One solution would be to implement the expressions given in [4], but this has not been done. So, we still reduce our geometrical domain to section from $X=2.1 \mathrm{~m}$ to $X=2.9 \mathrm{~m}$.

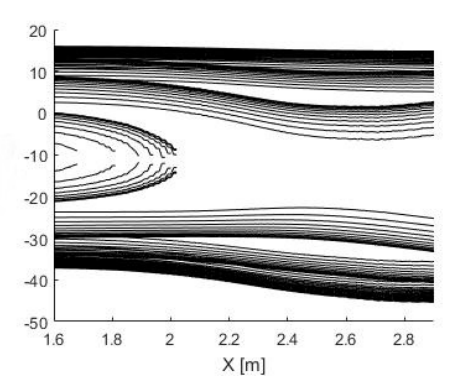

Fig. 6 Wave numbers of cut-on modes $[\mathrm{rad} / \mathrm{m}]$ as a function of bypass duct cross-section $X$, considering azimuthal modes order varying from -1 to 11 and initial Mach number 0.4 .

Before applying our deconvolution method, we have to define the position of the microphones of the virtual antenna. To do so, the condition number of matrix $\mathbf{G}^{\mathrm{H}} \mathbf{G}$ is minimized by using Matlab functions. We consider an infinite rigid annular duct of constant section, with outer radius $R_{\text {ext }}(2.4)$ and inner radius $R_{i n t}(2.4)$, and uniform constant flow of Mach number $M_{0}=0.4770$ (constant flow rate with Mach number equal to 0.4 at $X=1.3 \mathrm{~m}$ ). The first evaluation, 
made without constraint, gave an excessive antenna length. It has thus been constrained to a maximum length of $0.5 \mathrm{~m}$. Microphone locations obtained with the minimization process are represented in Figure 7 . Those microphones are projected on the bypass duct hub and casing. It would be interesting to take into account the variation of the modal basis along the $X$ axis. Nevertheless, the definition of microphone array would have been more complex as each iteration of the minimization process of the condition number of matrix $\mathbf{G}^{\mathrm{H}} \mathbf{G}$ changes the position of microphones as well as the modal basis.

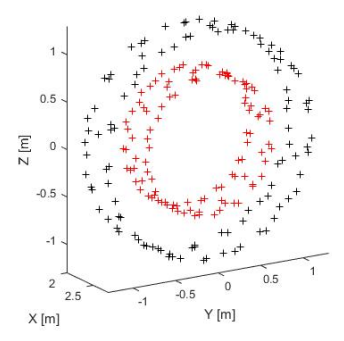

Fig. 7 Microphone antenna (218 microphones) obtained by minimization of the condition number of $\mathbf{G}^{\mathrm{H}} \mathbf{G}$, assuming a infinite rigid annular duct of constant section, with outer radius $R_{\text {ext }}(2.4)$ and inner radius $R_{\text {int }}(2.4)$, submitted to an uniform constant flow of Mach number 0.4770 .

The uncorrelated and correlated deconvolution methods ARMADA are applied, using the modal basis that has been used to define the microphone array, that is for infinite rigid annular duct with geometrical and flow conditions met at section $X=2.4 \mathrm{~m}$ when assuming a constant flow rate with Mach number equal to 0.4 at $X=1.3 \mathrm{~m}$. The restitution error obtained with this microphone array on simulated power cross spectral matrix of the amplitudes of the mode (see [1] for more details) is equal to $0.66 \%$. It is thus apply to data, and gives the mode amplitudes represented in Figure 8 The part of the data explained by the estimated matrix is greater than $74 \%$ under the correlated assumption. A constant flow rate with Mach number equal to 0.5 at $X=1.3 \mathrm{~m}$ is now considered. Unfortunately, the microphone antenna represented in Figure 7 is not relevant at $M_{0}=0.5$ : the restitution error on simulated power cross spectral matrix of the amplitudes of the mode is greater than $10 \%$. Thus, another specific microphone array is designed, composed by 218 microphones. The same analysis is done and gives results are represented in Figure 9
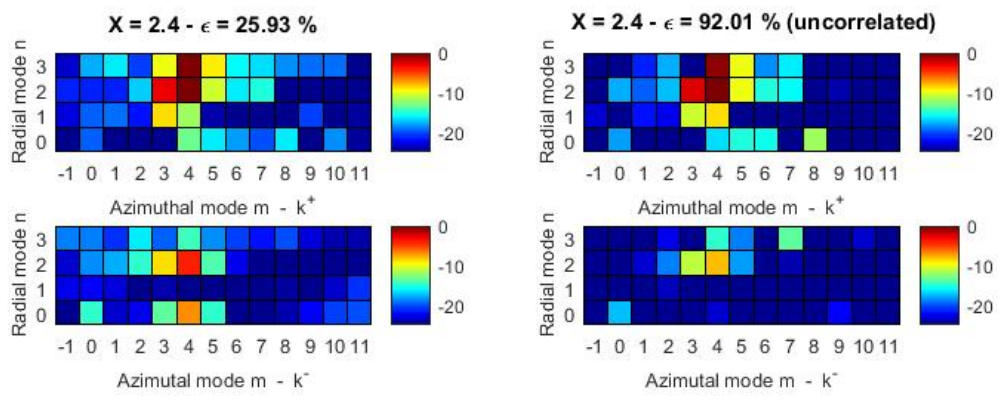

Fig. 8 Normalized mode amplitudes obtained by correlated (left) and uncorrelated (right) ARMADA on the bypass duct at BPF2, using the modal basis associated to infinite rigid annular duct with geometrical and flow conditions met at section $X=2.4 \mathrm{~m}$ and a constant flow rate determined by $M_{0}=0.4$ at $X=1.3 \mathrm{~m}$.

In both cases, the most energetic modes correspond to azimuthal mode $m=4$ and radial modes $n=2$ and 3 , together with azimuthal mode $m=3$ and radial mode $n=2$. Furthermore, those modes, represented in Figure 10 , are perfectly correlated. Modes propagating in the upstream direction $\left(k^{-}\right)$point out possible reflections at the end of the nacelle. The part of the data explained by the estimated matrix under the uncorrelated hypothesis is obviously reduced. Results are slightly different : amplitudes of upstream modes differ, and the amplitude of azimuthal mode $m=4$ and radial mode $n=3$ is under-estimated.

As our deconvolution method is based on several assumptions that are not fully verified, it is difficult to conclude on 

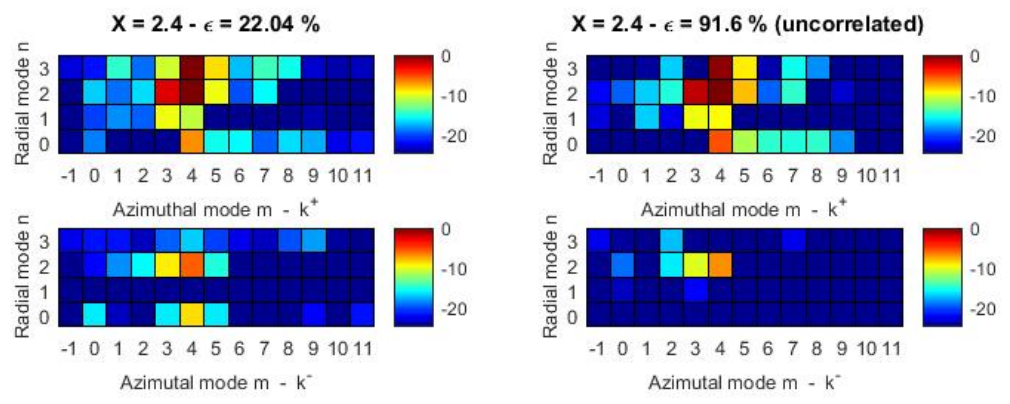

Fig. 9 Normalized mode amplitudes obtained by correlated (left) and uncorrelated (right) ARMADA on the bypass duct at BPF2, using the modal basis associated to infinite rigid annular duct with geometrical and flow conditions met at section $X=2.4 \mathbf{m}$ and a constant flow rate determined by $M_{0}=0.5$ at $X=1.3 \mathbf{~ m}$.
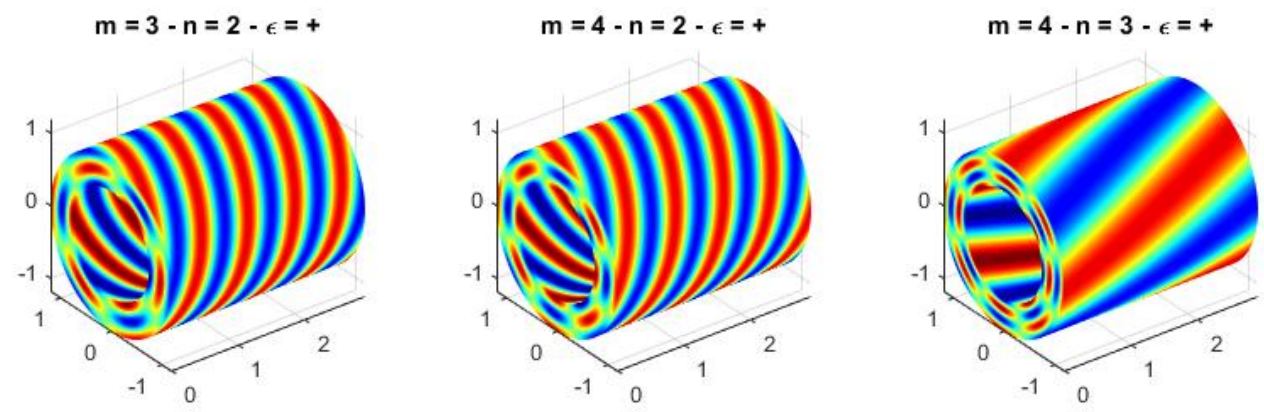

Fig. 10 The most energetic modes obtained after deconvolution by ARMADA.

the accuracy of the results. The best way would be to compare our results with the one obtained by another method. This information is currently not available. However, the absolute value of the Fourier transform of the acoustic pressure field can be reconstructed by using the estimated cross-spectral matrix of the amplitude of the modes and compare to data on the bypass duct hub and casing. The expression used is the following one :

$$
|\hat{p}(X, R, \theta)|^{2}=\sum_{i, j} \tilde{\mathbf{S}}_{i, j} \times U_{m_{i}, n_{i}}(R) U_{m_{j}, n_{j}}(R) e^{-\mathrm{i}\left(m_{i}-m_{j}\right) \theta} e^{-\mathrm{i}\left(k_{m_{i}, n_{i}}^{\varepsilon_{i}}-k_{m_{j}, n_{j}}^{\varepsilon_{j}}\right) X}
$$

with $\tilde{\mathbf{S}}$ the estimated power cross spectral matrix of the amplitude of the modes under the correlated modes assumption, considering a constant flow rate with $M_{0}=0.4$ and $M_{0}=0.5$ at $X=1.3 \mathrm{~m}$. Results obtained on the bypass duct hub and casing are represented in Figure 11. Data provided have been interpolated on a regular $(X, \theta)$-grid in order to conveniently compare the results. Angular step is set to $(2 \pi) / 180$ and space step is equal to $0.05 \mathrm{~m}$. The black frames highlight area ( $X$ from 1.6 to 2.02) where some modes, not included in the modal basis, go from cut-on to cut-off (see Figure 6). If the location of the maximum and minimum amplitudes are well retrieved, the wavelength of the texture observed on the data seems to be longer than the one obtained with $M_{0}=0.4$. Those observed with $M_{0}=0.5$ seems to be even shorter. However, using only data obtained on the wall, ONERA's deconvolution method succeeds in providing a good representation of the acoustic field in the bypass duct.

\section{Conclusion}

In this study, the modal content of the acoustic pressure field computed by CFD [2] on the take-off side-line case at BPF2 is analysed. Only data provided on the wall of the inlet duct casing and bypass duct hub and casing are used, as it would be for measurements obtained by flush mounted microphones (non-intrusive measurements).

As a first step, the azimuthal mode content of the acoustic pressure field, using an azimuthal Fourier transform, is determined. The rotor-locked mode is dominant in the inlet duct casing, closed to the fan, but adjacent modes appear 

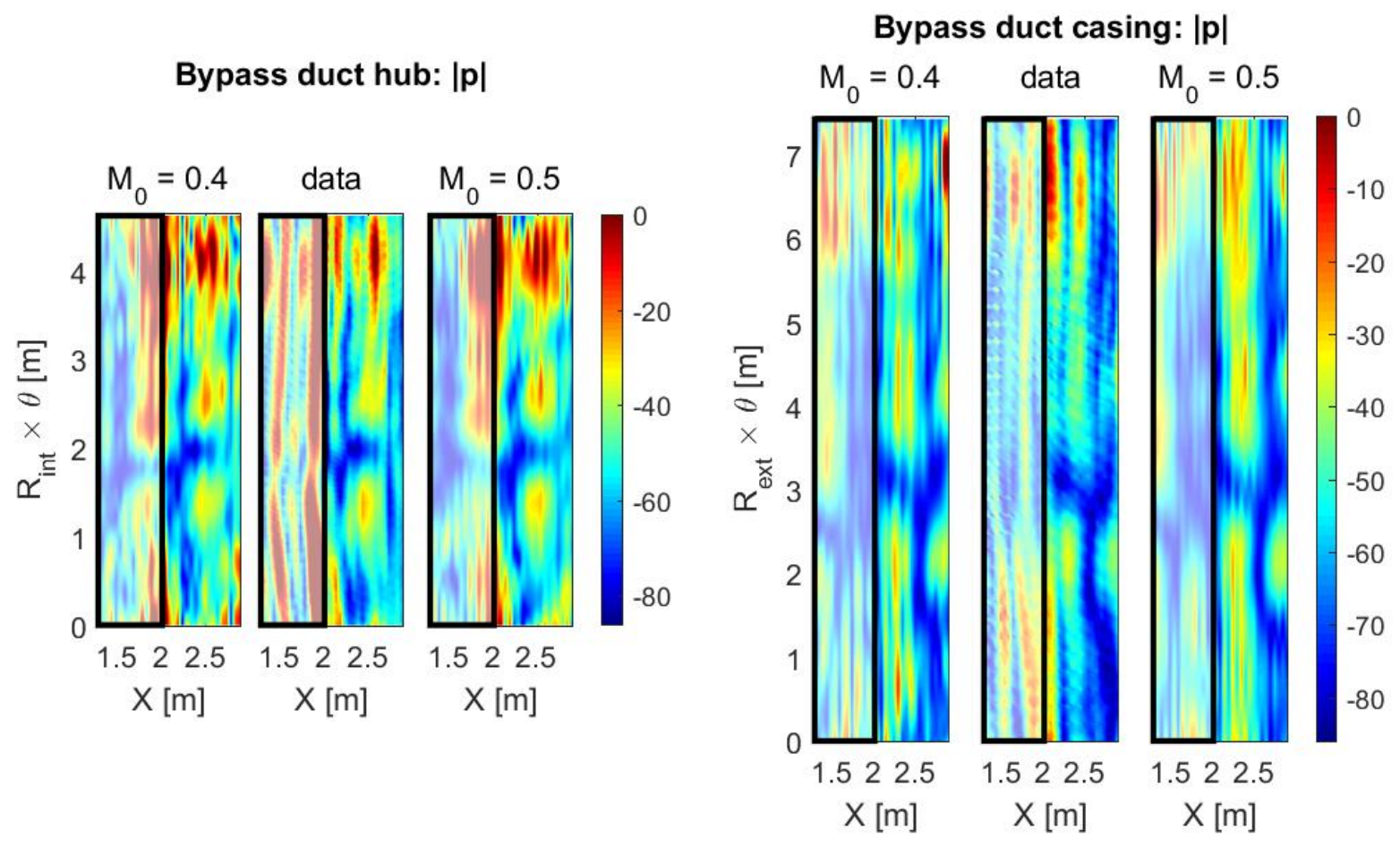

Fig. 11 Absolute value of the Fourier transform of the pressure field reconstructed on the bypass duct hub surface (left) and duct casing surface (right).

and the azimuthal mode content of the sound pressure field sharply vary in the vicinity of the inlet. In the bypass duct, the Tyler-Sofrin interaction and adjacent modes prevail all along the $\mathrm{X}$-axis.

As a second step, we focus on data provided on bypass duct and restrict ourselves to section from $X=1.57 \mathrm{~m}$ to $2.9 \mathrm{~m}$, where azimuthal modes from -1 to 11 explain more than $98 \%$ of the total sound pressure level. An azimuthal mode deconvolution method is applied, assuming that modes are correlated or not. The amplitude of the most energetic azimuthal modes are validated by comparison with the one obtained by azimuthal Fourier transform. However, the part of the power cross spectral matrix of the measurements that is explained with the azimuthal Fourier transform or the uncorrelated azimuthal deconvolution method is quite low (10\% to 65\%), whereas results obtained with the azimuthal deconvolution method under correlated modes assumption explain the most part of it $(80 \%$ to $90 \%)$. Those results encourage us to consider that azimuthal modes are correlated.

As a third step, we apply the azimuthal and radial mode deconvolution method ARMADA [1], assuming that azimuthal as well as radial modes are correlated. Indeed, our modal analysis method assumes a constant section annular duct and uniform flow while the configuration studied here implies a complex geometry of the nacelle, and a highly variable Mach number through the fan channel. Geometrical and flow conditions met at section $X=2.4 \mathrm{~m}$ when assuming a constant flow rate are used to define the mode basis. As the Mach number through the fan duct is not precisely known, values of $M_{0}=0.4$ and $M_{0}=0.5$ are taken. The microphone antenna is defined by minimization of the condition number of matrix $\mathbf{G}^{\mathrm{H}} \mathbf{G}$, where $\mathbf{G}$ denotes the propagation matrix. The part of the data explained by the estimated matrix is greater than $74 \%$. With both Mach number, the most energetic modes correspond to azimuthal mode $m=4$ and radial modes $n=2$ and 3, together with azimuthal mode $m=3$ and radial mode $n=2$; they are perfectly correlated. Reflections may occur at the end of the nacelle as upstream modes have non negligible amplitudes.

As our deconvolution method is based on several assumptions that are not fully verified, it is difficult to conclude on our results accuracy. To have an idea on this point, we finally compute the absolute value of the Fourier transform of the acoustic pressure field on the bypass duct hub and casing by using the estimated cross spectral matrix of the amplitude of the modes. The shape of the data is retrieved. To conclude, using only data obtained on the wall, ONERA's deconvolution method succeeds in providing a good representation of the acoustic field in the bypass duct. 


\section{Acknowledgement}

ONERA carried out this study in the framework of the ASPIRE project which has received funding from the European Union's H2020 program for the Clean Sky Joint Technology Initiative under grant agreement number 681856. The author would like to thank Mr Laban and Mr Brouwer, from NLR, for the data and email exchanges, as well as Mr Davy and Mr Polacsek, from ONERA, for fruitful scientific discussions.

\section{References}

[1] Fauqueux, S., and Davy, R., "Modal deconvolution method in a finite circular duct, using flush-mounted microphones," 24th AIAA/CEAS Aeroacoustics Conference, AIAA, Atlanta, Georgia, 2018. doi:10.2514/6.2018-3927.

[2] Laban, M., Kok, J., and Brouwer, H., "CFD/CAA Analysis of UHBR Engine Tonal Noise," 24th AIAA/CEAS Aeroacoustics Conference, AIAA, Atlanta, Georgia, 2018. doi:10.2514/6.2018-3780.

[3] Rienstra, S., and Hirschberg, A., An Introduction to Acoustics, Eindhoven University of Technology, 2017. https://www . win. tue.nl/ sjoerdr/papers/boek.pdf

[4] Rienstra, S., "Sound transmission in slowly varying circular and annular lined ducts with flow," Journal of Fluid Mechanics, Vol. 380, 1999, pp. 279, 296. 